\title{
Prognostic Significance of Pigment Epithelium-Derived Factor Expression in Patients with Non-Small-Cell-Lung Cancer
}

\author{
Alexander Emmert ${ }^{1 * \#}$, Angelika Oellerich ${ }^{1 \#, ~ L a s z l o ~ F u ̈ z e s i 2 ~}{ }^{2}$, Regina Waldmann-Beushausen', \\ Friedrich A. Schöndube ${ }^{1}$, Hanibal Bohnenberger ${ }^{2 \dagger}$, Bernhard C. Danner ${ }^{1 \dagger}$ \\ ${ }^{1}$ Department of Thoracic and Cardiovascular Surgery, University Medical Center Göttingen, Göttingen, Germany \\ ${ }^{2}$ Institute of Pathology, University Medical Center Göttingen, Göttingen, Germany \\ Email: *alexander.emmert@med.uni-goettingen.de
}

How to cite this paper: Emmert, A., Oellerich, A., Füzesi, L., Waldmann-Beushausen, R., Schöndube, F.A., Bohnenberger, H. and Danner, B.C. (2016) Prognostic Significance of Pigment Epithelium-Derived Factor Expression in Patients with Non-Small-CellLung Cancer. Advances in Lung Cancer, 5, 30-38.

http://dx.doi.org/10.4236/alc.2016.53004

Received: August 16, 2016

Accepted: September 19, 2016

Published: September 22, 2016

Copyright $\odot 2016$ by authors and Scientific Research Publishing Inc. This work is licensed under the Creative Commons Attribution International License (CC BY 4.0).

http://creativecommons.org/licenses/by/4.0/

\begin{abstract}
The aims of this study were to examine prognostic significance of pigment epithelium-derived factor (PEDF) in patients with stage IA-IIIB non-small cell lung cancer (NSCLC). Using immunohistochemistry and multivariate analysis, we set out to investigate whether PEDF expression could provide prognostic information in NSCLC in a cohort of 69 patients who had undergone radical resection for NSCLC. The correlation between PEDF and the clinical pathological features of stage I-III NSCLC after radical surgery were analyzed as well as influence on long term survival. No correlation between PEDF intensity, PEDF area or PEDF area index and clinic opathologic parameters was seen. PEDF values showed a slight correlation to the tumor stage. There was a significant negative correlation $(T=-0.288, p=0.002)$ between pathologic $\mathrm{T}$-stage and median PEDF area and vice versa a positive correlation $(\mathrm{T}=$ $0.227, \mathrm{p}=0.016)$ with median PEDF intensity. We could not detect any correlation between PEDF and long term survival. For PEDF analysis, there was only a slight correlation between expression and T-stage of the tumor.
\end{abstract}

\section{Keywords}

NSCLC, PEDF, Neoangiogenesis

\section{Background}

Non-small-cell lung cancer (NSCLC) is one of the most common cancers worldwide

\#Alexander Emmert and Angelika Oellerich contributed equally to this work.

${ }^{\dagger}$ Hanibal Bohnenberger and Bernhard C. Danner contributed equally to this work. 
and the leading cause of cancer related death in several countries. With the recent advances in screening, diagnosis, and treatment of this disease, guidelines are constantly being reviewed to provide the best in detection and therapy. Currently, the new TNM classification for lung cancer is being proposed by the International Association for the Study of Lung Cancer (IASLC), and the 8th edition of TNM staging system is expected to be released in 2016 [1]. However, especially for early stage NSCLC, prognostic biomarkers are still lacking.

NSCLC is characterized by uncontrolled growth and spread of abnormal cells and by tumor neoangiogenesis [2] [3]. Under physiological conditions, neoangiogenesis is the formation of new capillaries and blood vessels by growth and differentiation from existing ones. It occurs mainly during embryonic development, but also at wound sites [4]. In recent years, therapies selectively targeting neoangiogenesis pathways, such as VEGFR have both provided a better understanding of NSCLC and have been investigated as prognostic factors or targets for individualized therapy [5]-[7], but have not led to a significant therapy improvement.

A potentially significant molecule, in understanding NSCLC neoangiogenesis is Pigment Epithelium-DerivedFactor (PEDF), also known as early population doubling level cDNA-1. PEDF is an endogenous $50-\mathrm{kDa}$ glycoprotein that is first shown to be capable of inducing differentiation in Y-79 retinoblastoma cells [8]-[11]. Among its other functions, it inhibits the proliferation and migration of endothelial cells toward various angiogenic inducers and thereby suppresses angiogenesis [8]-[11]. In the context of tumors, PEDF exhibits an impressive tumor growth-suppressing activity by targeting tumor vasculature and tumor cells per se. Initially, the antitumor activity of PEDF was proven to be attributable to its antiangiogenic property [12] [13]. Furthermore, PEDF expression is significantly reduced in a wide range of tumor types, including pancreatic adenocarcinoma, glioblastoma and ovarian carcinoma [14] [15] and its recovered expression in these tumors delays the onset of primary tumors and decreases metastasis [14] [15].

Using immunohistochemistry and multivariate analysis, we set out to investigate whether PEDF expression could provide prognostic information in NSCLC patients. The correlation between PEDF and the clinical pathological features of stage I - III NSCLC after radical surgery were analysed.

\section{Methods}

\subsection{Patient Inclusion and Tissue Samples}

A total of 69 patients with a stage IA - III NSCLC (either adenocarcinoma ADC or squamous cell carcinoma SCC), who underwent radical surgical resection at the thoracic surgery department of Thoracic and Cardiovascular Surgery of the Göttingen University Medical Center were enrolled in this retrospective study. Exclusion criteria were NSCLC stage IV and unrespectable tumor. The local ethical committee approved this study (\#1-2-08). Preoperative interdisciplinary tumor board meetings were held and proposed curative resection surgery for each patient included. None of the patients 
had received prior chemo or radiation therapy. No other previous or concomitant primary cancer was present. All procedures were conducted in accordance with institutional, state and federal guidelines.

WHO classification was used for histological assessment of each specimen and pathological staging of each tumor was determined using the renewed TNM staging system for NSCLC [8]. Histological types of lung cancer included: ADC in 27 patients (39.1\%), and SCC in 42 patients (60.9\%). Table 1 summarizes further clinical characteristics.

All immunostained sections were reviewed by two investigators who had no knowledge of the patients' clinical status. A structured follow-up questionnaire was sent to oncologists, pulmonary physicians and general practitioners.

\subsection{Tumor PEDF Immunohistochemistry}

Formalin fixed and paraffin-embedded primary lung tumor tissue was selected by pathologists to allow clear definition of tumor tissue areas on haematoxylin-eosin (H\&E) stained sections. A two-step indirect streptavidin-biotin technique was used for further

Table 1. Clinical characteristics of included lung cancer patients.

\begin{tabular}{|c|c|c|}
\hline Histology & Adenocarcinoma $(n=27)$ & Squamous cell carcinoma $(n=42)$ \\
\hline Age (years) & $63.55 \pm 10.8$ & $66.2 \pm 7.7$ \\
\hline \multirow{2}{*}{ Gender } & 20 male $(74.1 \%)$ & 41 male $(97.6 \%)$ \\
\hline & 7 female $(25.9 \%)$ & 71 female $(2.4 \%)$ \\
\hline \multirow{3}{*}{ Operation type } & Pneumectomy: 3 (11.1\%) & Pneumectomy: 10 (23.8\%) \\
\hline & Lobectomy: 22 (81.5\%) & Lobectomy: 27 (64.3\%) \\
\hline & Bilobectomy: 2 (7.4\%) & Bilobectomy: 5 (11.9\%) \\
\hline Lymphnodes resected (n) & $17.8 \pm 6.8$ & $20.9 \pm 8.6$ \\
\hline \multirow{6}{*}{ Pathologic Stage } & IA: $3(11.1 \%)$ & IA: $3(7.1 \%)$ \\
\hline & IB: $13(48.1 \%)$ & IB: $12(28.6 \%)$ \\
\hline & IIA: $1(3.7 \%)$ & IIA: $9(21.4 \%)$ \\
\hline & IIB: $4(14.8 \%)$ & IIB: $8(19.0 \%)$ \\
\hline & IIIA: $6(22.2 \%)$ & IIIA: $9(21.4 \%)$ \\
\hline & & IIIB: $1(2.4 \%)$ \\
\hline \multirow{6}{*}{ T-Status } & T1a: $17(18.5 \%)$ & T1a: $3(7.1 \%)$ \\
\hline & T1b: $6(22.2 \%)$ & T1b: 7 (16.7\%) \\
\hline & T2a: $10(37.0 \%)$ & T2a: $13(31.0 \%)$ \\
\hline & T2b: $4(14.8 \%)$ & T2b: 9 (21.4\%) \\
\hline & T3: $2(7.4 \%)$ & T3: 7 (16.7\%) \\
\hline & & T4: $3(7.1 \%)$ \\
\hline \multirow{4}{*}{$\mathrm{N}$-Status } & N0: 17 (63\%) & N0: 27 (64.3\%) \\
\hline & $\mathrm{N} 1: 16(14.8 \%)$ & N1: $9(21.4 \%)$ \\
\hline & $\mathrm{N} 2: 6(22.2 \%)$ & N2: $5(11.9 \%)$ \\
\hline & & N3: $1(2.4 \%)$ \\
\hline \multirow{3}{*}{ Grading } & $1^{\circ}$ Grade: $1(3.7 \%)$ & \\
\hline & $2^{\circ}$ Grade: $17(63.0 \%)$ & $2^{\circ}$ Grade: $28(66.7 \%)$ \\
\hline & $3^{\circ}$ Grade: $9(33.3 \%)$ & $3^{\circ}$ Grade: $14(33.3 \%)$ \\
\hline Mean follow up time (month) & $35 \pm 27.9$ & $51.5 \pm 35.9$ \\
\hline
\end{tabular}


immunohistochemical staining. Following deparaffinisation and rehydration the peroxidase was rinsed with $3 \% \mathrm{H}_{2} \mathrm{O}_{2}$ (S2023, Dako, Hamburg, Germany) and samples were then washed with Tris buffered saline (TBS). Staining PEDF was carried out using monoclonal antibody JC70A (Dako, Hamburg, Germany) and monoclonal antibody SerpinF1/PEDF (R\&B, Abingdon, Great Britain) respectively.

\subsection{Evaluation of PEDF-Staining}

Regions of interest were determined by the hot spot method and detection of vital tumour tissue and cancer cells by the H\&E stained sections. These regions were analyzed at a magnification of $\times 25$ by two separate investigators. Signal intensity was measured by semi quantitative scale $(1,1+, 2-, 2,2+, 3-, 3,3+)$ and median values were determined. As PEDF signalling appeared in spot like forms, a PEDF signalling area measurement was undertaken in $\mu^{2}$. A product of the intensity index and area of PEDF was calculated (Area-intensity index).

\subsection{Statistical Analysis}

The following variables were examined and analyzed with regard to the findings of immunohistochemistry: gender, histopathology, UICC and pT stage, lymph node status, and grading. Categorical variables were expressed as proportions (\%) or number (n) and continuous variables as mean \pm standard deviation (SD) and median with range. Long-term follow-up (survival or lung cancer related death at 90 days after surgery) was calculated (using the Kaplan-Meier method). Differences between groups were calculated and compared (using the log-rank test). Cox Regression $t$ influence of clinicopathologic parameters analysis was used. Association analysis for linear correlation of median PEDF values was given as Pearson's correlation coefficient, whereas Kendall tau-b correlation coefficient $\mathrm{T}$ was provided for correlation with clinicopathologic parameters. Results were considered significant for $\mathrm{p}$ values below 0.05 . Long term follows up, defined as survival or death after 90 days postoperative could be fully completed. To test the influence of PEDF expression on long-term survival, all patients with known lung cancer death or living were studied. Statistical modelling and testing were performed with SPSS ${ }^{\oplus}$ statistical software (Release 15.0.0).

\section{Results}

A total of 69 patients with available NSCLC tumor tissue blocks were included in the analysis. During a median follow-up of 48 months, patients were clinically evaluated and were referred for radiological assessment as per clinical indications. The sociodemographic and clinical characteristics of the patients are described in Table 1. At the time of last follow-up 25 patients were alive (36.2\%). Overall median survival time was 45 month. Lung cancer related death occurred in 33 patients (47.8\%); for unknown reasons in $6(8.7 \%)$, due to a secondary tumor in $1(1.5 \%)$, and for other non-tumorous reasons in 4 patients (5.8\%).

In PEDF staining, the mean intensity of signalling was $2.18 \pm 0.50$ (median 2.22, 
range 1.1 - 3.2). The mean PEDF area was $1012 \pm 1285 \mathrm{~mm}^{2}$ (median $682 \mathrm{~mm}^{2}$, range 0 - $4617 \mathrm{~mm}^{2}$ ), and the area intensity index was mean $2142 \pm 2731$, median 1437 (range 0 - 8601). PEDF staining did not correlate with gender, histopathology, lymph node status or grading. Nevertheless, there was a significant difference of median PEDF intensity values between UICC stage $(T=0.243, \mathrm{p}=0.010)$, as well as a slight but positive correlation $(\mathrm{T}=0.240, \mathrm{p}=0.010)$ to $\mathrm{pT}$ stage (Figure 1 ). Vice versa, the median PEDF area showed a negative correlation with UICC stage $(\mathrm{T}=-0.224, \mathrm{p}=0.013)$ and $\mathrm{pT}$ stage $(\mathrm{T}=-0.305, \mathrm{p}<0.01$; Figure 2$)$.

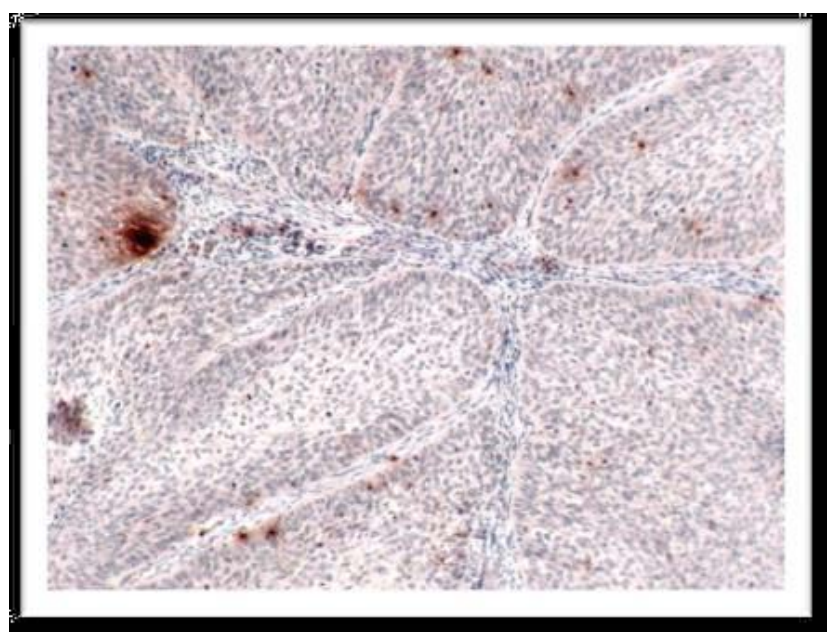

Figure 1. Staining of PEDF was carried out using monoclonal antibody monoclonal antibody SerpinF1/PEDF respectively. Regions of interest were determined by the hot spot method outlined above and detection of vital tumour tissue and cancer cells by the H\&E stained sections. These regions were analysed at a magnification of $25 \times$.

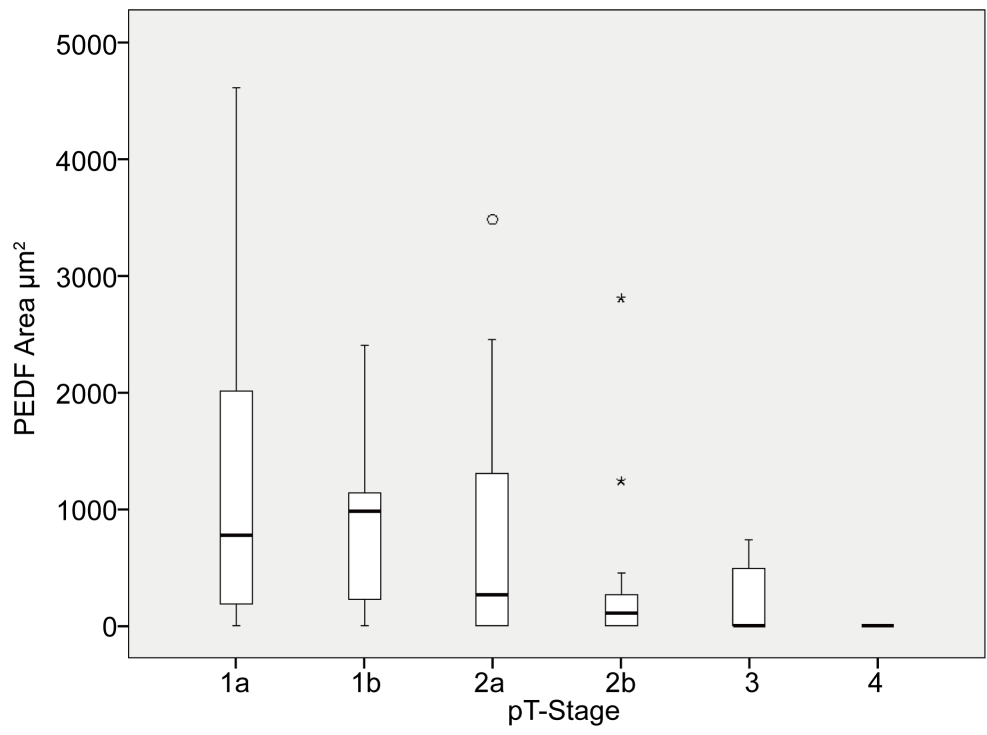

Figure 2. The median PEDF area showed a negative correlation with UICC stage $(\mathrm{T}=-0.224, \mathrm{p}=0.013)$ and $\mathrm{pT}$-stage $(\mathrm{T}=-0.305, \mathrm{p}<0.01)$. 


\section{Discussion}

In this retrospective study we evaluated the prognostic value PEDF in patients with resected NSCLC of stage IA-III.

In 1989, Folkman hypothesized that tumor growth is dependent on successful angiogenesis and that methods that inhibit angiogenesis could be integrated into tumor therapy [2]. Neoangiogenesis is a complex phenomenon and solid tumors cannot grow beyond $1-2 \mathrm{~mm}$ in diameter without neoangiogenesis [16]. Expanding evidence suggests that neoangiogenesis is also an integral part of tumor cell dissemination and propensity for metastasize [2] [3]. Therefore detailed assessment of neoangiogenesis in tumors could not only lead to the development of new improved anti-angiogenic therapies, but also provide information that could help stratify patients into different treatment regimens and potentially predict response to chemotherapy [5]-[7]. Especially in patients with early stage lung cancer, reliable prognostic factors for risk stratification and decision upon adjuvant chemotherapy still need to be clinically established [17] [18].

Although, studies on the relationship of PEDF expression (particularly in NSCLC) are few in number more standardized methods of assessing neoangiogenesis should be agreed upon, if studies are to be compared and reliable conclusions drawn.

We looked at PEDF, which acts directly on endothelial cells and can halt new vessel formation by inducing apoptosis in endothelial cells stimulated during new vessel formation. PEDF inhibits the proliferation and migration of endothelial cells and is perhaps the most potent anti-angiogenic factor to be identified [19]. It has been shown to be reduced in NSCLC and gene therapy research in other forms of cancer e.g. DPEDF inhibiting human pancreatic cancer cells in mice has been promising and may provide a novel approach for treatment of pancreatic adenocarcinoma [20] [21]. We sought to build on these previous findings and investigate PEDF as a prognostic factor in NSCLC. After analysing tumour samples obtained in surgery, we found no correlation between PEDF staining and gender, histopathology, lymph node status or tumour grading in NSCLC patients. However, similarly to a previous study with 91 patients, a significant positive correlation of PEDF intensity values and pathologic T-stage was observed. PEDF expression has also been shown to be related to TNM staging and tumor size and a screening for expression of PEDF in liver metastasis (from ductal pancreatic adenocarcinoma) identified PEDF expression as an independent positive prognostic factor-in PEDF positive samples, patient survival was significantly longer than when PEDF was not expressed [22]. Whilst our study in NSCLC, did show a correlation with pathologic T-stage and PEDF expression, no actual influence of PEDF on survival was observed.

Nonetheless, although PEDF expression may not appear to be useful indicator of prognosis, it still could be of clinical importance. The past few years have seen rapid development and improvement of tumor inhibitors, and an increasing body of evidence suggests that selective inhibitors of growth factors (e.g. EGFR) provide useful therapeutic agents, particularly in advanced NSCLC. 


\section{Conclusion}

In conclusion, we found that PEDF (a potential antiangiogenic factor) has no significance with long-term survival. Significant limitations to this study include that it is a retrospective study and that only patients who have underwent surgery are included. Additionally, although our findings may appear surprising or even paradoxical, previous studies on MVD have also been controversial and unequivocal. Nevertheless, even though studies on the subject continue to yield seemingly contradictory results, the role of this factor in antitumor therapy should continue to be researched and perhaps standardised prospective studies will elucidate matters.

\section{Conflict of Interest}

The authors declare that they have no conflict of interest.

\section{Author's Contributions}

$\mathrm{AE}, \mathrm{BCD}$ and FAS conceived of the study and participated in its design. AO participated in the design of the study and contributed to the immunohistochemical analysis. LF, HB and RWB participated in the interpretation of the pathologic data. AE and BCD drafted the manuscript. All authors read and approved the final manuscript.

\section{Acknowledgements}

We acknowledge support by the German Research Foundation and the Open Access Publication Funds of the Göttingen University.

Hanibal Bohnenberger was supported by the Else-Kröner-Fresenius-Foundation.

\section{References}

[1] El Masri, J., Ren, S. and Zhang, J. (2016) Getting Familiar with the Forthcoming Eighth Edition of TNM Classification of Lung Cancer: From the T to N and M Descriptors. Annals of Translational Medicine, 4, 67.

[2] Hanahan, D. and Folkman, J. (1996) Patterns and Emerging Mechanisms of the Angiogenic Switch during Tumorigenesis. Cell, 86, 353-364. http://dx.doi.org/10.1016/S0092-8674(00)80108-7

[3] Folkman, J. (1989) What Is the Evidence That Tumors Are Angiogenesis Dependent? Journal of the National Cancer Institute, 82, 4-6.

[4] Bodnar, R.J. (2015) Chemokine Regulation of Angiogenesis during Wound Healing. Advances in Wound Care (New Rochelle), 4, 641-650.

http://dx.doi.org/10.1089/wound.2014.0594

[5] Pao, W. and Girard, N. (2011) New Driver Mutations in Non-Small-Cell Lung Cancer. The Lancet Oncology, 12, 175-180. http://dx.doi.org/10.1016/S1470-2045(10)70087-5

[6] Fu, L., Shi, K., Wang, J., Chen, W., Shi, D., Tian, Y., Guo, W., Yu, W., Xiao, X., Kang, T., Wang, S., Huang, W. and Deng, W. (2014) TFAP2B Overexpression Contributes to Tumor Growth and a Poor Prognosis of Human Lung Adenocarcinoma through Modulation of ERK and VEGF/PEDF Signaling. Molecular Cancer, 13, 89.

http://dx.doi.org/10.1186/1476-4598-13-89 
[7] Villaruz, L.C. and Socinski, M.A. (2015) The Role of Anti-Angiogenesis in Non-Small-Cell Lung Cancer: An Update. Current Oncology Reports, 17, 26.

http://dx.doi.org/10.1007/s11912-015-0448-y

[8] Pignolo, R.J., Cristofalo, V.J. and Rotenberg, M.O. (1993) Senescent WI-38 Cells Fail to Express EPC-1, a Gene Induced in Young Cells upon Entry into the G0 State. The Journal of Biological Chemistry, 268, 8949-8957.

[9] Pignolo, R.J., Rotenberg, M.O. and Cristofalo, V.J. (1995) Analysis of EPC-1 Growth State-Dependent Expression, Specificity, and Conservation of Related Sequences. Journal of Cellular Physiology, 162, 110-118. http://dx.doi.org/10.1002/jcp.1041620113

[10] Dawson, D.W., Volpert, O.V., Gillis, P., Crawford, S.E., Xu, H., Benedict, W. and Bouck, N.P. (1999) Pigment Epithelium-Derived Factor: A Potent Inhibitor of Angiogenesis. Science, 285, 245-248. http://dx.doi.org/10.1126/science.285.5425.245

[11] Steele, F.R., Chader, G.J., Johnson, L.V. and Tombran-Tink, J. (1993) Pigment EpitheliumDerived Factor: Neurotrophic Activity and Identification as a Member of the Serine Protease Inhibitor Gene Family. Proceedings of the National Academy of Sciences of the United States of America, 90, 1526-1530. http://dx.doi.org/10.1073/pnas.90.4.1526

[12] Broadhead, M.L., Dass, C.R. and Choong, P.F. (2009) In Vitro and in Vivo Biological Activity of PEDF against a Range of Tumors. Expert Opinion on Therapeutic Targets, 13, 14291438. http://dx.doi.org/10.1517/14728220903307475

[13] Li, L., Yao, Y.C., Fang, S.H., Ma, C.Q., Cen, Y., Xu, Z.M., Dai, Z.Y., Li, C., Li, S., Zhang, T., Hong, H.H., Qi, W.W., Zhou, T., Li, C.Y., Yang, X. and Gao, G.Q. (2014) Pigment Epithelial-Derived Factor (PEDF)-Triggered Lung Cancer Cell Apoptosis Relies on p53 ProteinDriven Fas Ligand (Fas-L) Up-Regulation and Fas Protein Cell Surface Translocation. The Journal of Biological Chemistry, 289, 30785-30799. http://dx.doi.org/10.1074/jbc.M114.590000

[14] Hoshina, D., Abe, R., Yamagishi, S.I. and Shimizu, H. (2010) The Role of PEDF in Tumor Growth and Metastasis. Current Molecular Medicine, 10, 292-295. http://dx.doi.org/10.2174/156652410791065327

[15] Zhou, D., Zhang, M., Xu, P., Yu, Y., Ye, G., Zhang, L. and Wu, A. (2016) Expression of Pigment Epithelium-Derived Factor Is Associated with a Good Prognosis and Is Correlated with Epithelial-Mesenchymal Transition-Related Genes in Infiltrating Ductal Breast Carcinoma. Oncology Letters, 11, 116-124.

[16] Hillen, F. and Griffioen, A.W. (2007) Tumour Vascularization: Sprouting Angiogenesis and Beyond. Cancer and Metastasis Reviews, 26, 489-502. http://dx.doi.org/10.1007/s10555-007-9094-7

[17] Zhou, D., Cheng, S.Q., Ji, H.F., Wang, J.S., Xu, H.T., Zhang, G.Q. and Pang, D. (2010) Evaluation of Protein Pigment Epithelium-Derived Factor (PEDF) and Microvessel Density (MVD) as Prognostic Indicators in Breast Cancer. Journal of Cancer Research and Clinical Oncology, 136, 1719-1727. http://dx.doi.org/10.1007/s00432-010-0830-y

[18] Massuti, B., Sanchez, J.M., Hernando-Trancho, F., Karachaliou, N. and Rosell, R. (2013) Are We Ready to Use Biomarkers for Staging, Prognosis and Treatment Selection in Early-Stage Non-Small-Cell Lung Cancer? Translational Lung Cancer Research, 2, 208-221.

[19] Chandolu, V. and Dass, C.R. (2012) Cell and Molecular Biology Underpinning the Effects of PEDF on Cancers in General and Osteosarcoma in Particular. Journal of Biomedicine and Biotechnology, 2012, Article ID: 740295. http://dx.doi.org/10.1155/2012/740295

[20] Shi, H.S., Yang, L.P., Wei, W., Su, X.Q., Li, X.P., Li, M., Luo, S.T., Zhang, H.L., Lu, L., Mao, Y.Q., Kan, B. and Yang, L. (2013) Systemically Administered Liposome-Encapsulated 
Ad-PEDF Potentiates the Anti-Cancer Effects in Mouse Lung Metastasis Melanoma. Journal of Translational Medicine, 11, 86. http://dx.doi.org/10.1186/1479-5876-11-86

[21] Hase, R., Miyamoto, M., Uehara, H., Kadoya, M., Ebihara, Y., Murakami, Y., Takahashi, R., Mega, S., Li, L., Shichinohe, T., Kawarda, Y. and Kondo, S. (2005) Pigment Epithelium-Derived Factor Gene Therapy Inhibits Human Pancreatic Cancer in Mice. Clinical Cancer Research, 11, 8737-8744. http://dx.doi.org/10.1158/1078-0432.CCR-05-1323

[22] Uehara, H., Miyamoto, M., Kato, K., Ebihara, Y., Kaneko, H., Hashimoto, H., Murakami, Y., Hase, R., Takahashi, R., Mega, S., Shichinohe, T., Kawarady, Y., Itoh, T., Okushiba, S., Kondo, S. and Katoh, H. (2004) Expression of Pigment Epithelium-Derived Factor Decreases Liver Metastasis and Correlates with Favorable Prognosis for Patients with Ductal Pancreatic Adenocarcinoma. Cancer Research, 64, 3533-3537.

http://dx.doi.org/10.1158/0008-5472.CAN-03-3725

\section{Abbreviations}

H\&E: Hematoxylin-Eosin Staining

IHC: Immunohistochemistry

NSCLC: Non-Small Cell Lung Cancer

MVD: Microvessel Density

PECAM-1: Platelet Endothelial Cell Adhesion Molecule-1

SD: Standard Deviation, PEDF

Submit or recommend next manuscript to SCIRP and we will provide best service for you:

Accepting pre-submission inquiries through Email, Facebook, LinkedIn, Twitter, etc.

A wide selection of journals (inclusive of 9 subjects, more than 200 journals)

Providing 24-hour high-quality service

User-friendly online submission system

Fair and swift peer-review system

Efficient typesetting and proofreading procedure

Display of the result of downloads and visits, as well as the number of cited articles

Maximum dissemination of your research work

Submit your manuscript at: http://papersubmission.scirp.org/

Or contact alc@scirp.org 